\title{
Ischaemic stroke and influenza A H1N1 vaccination: a case report
}

Yi-Pin Lin' ${ }^{1}$ Tzu-Hui Li ${ }^{1}$, Wei-Hsi Chen ${ }^{1,2}$

\begin{abstract}
1Department of Neurology, Chang Gung Memorial Hospital-Kaohsiung Medical Center, and College of Medicine, Chang Gung University, Kaohsiung, Taiwan

${ }^{2}$ Graduate Institute of Science and Technology Law, National Kaohsiung First University of Science and Technology, Kaohsiung, Taiwan
\end{abstract}

Submitted: 23 April 2010

Accepted: 7 August 2010

Arch Med Sci 2011; 7, 2: 345-348

DOI: 10.5114/aoms.2011.22090

Copyright (C) 2011 Termedia \& Banach

\section{Abstract}

We report a 75-year-old male patient who suffered posterior circulation ischaemia after influenza A/H1N1 vaccination. Vaccination provokes a variable magnitude of inflammatory and immunological response that modifies the risk for ischaemic stroke. Whereas a causal relation between vaccination and ischaemic stroke is still unsettled, an inflammatory/immunological response after vaccination may trigger thrombosis superimposing a pre-existing prothrombotic state. Careful monitoring is strongly suggested for individuals who received H1N1 vaccine, especially those with high ischaemic stroke risk.

Key words: influenza A/H1N1, vaccine, ischaemic stroke, cerebellum, risk factor, thrombosis.

\section{Introduction}

In contrast to a variety of established traditional and emerging risk factors, only a few reports concern ischaemic stroke occurrence after vaccination [1-5]. This rarity of association is criticized for the reliability and validity of a causal relation between vascular events and vaccination, particularly when the frequency of ischaemic stroke and myocardial infarction does not increase shortly after vaccination with the influenza vaccine [6]. Ambivalence arises when vaccination causes an inflammatory reaction that emphasizes an emerging stroke risk factor on one hand, and a low frequency of ischaemic stroke occurs after vaccination on the other hand.

In this study, we report a patient who suffered ischaemic stroke shortly after influenza $\mathrm{A} 1 / \mathrm{H} 1 \mathrm{~N} 1$ vaccination. In order to elucidate whether a risk factor is present for ischaemic stroke in vaccination, we extracted data from the Vaccine Adverse Event Reporting System (VAERS), Department of Health and Human Services, United States of America [7], and reviewed previous reports of vaccination-related ischaemic stroke.

\section{Case report}

A 75-year-old man received influenza A/H1N1 vaccination at 10 a.m. on one day. He then went home by motorcycle, cooked and finished lunch, completed house work, and then napped at noon. He usually woke up

\author{
Corresponding author: \\ Dr. Wei-Hsi Chen \\ Department of Neurology \\ Chang Gung Memorial \\ Hospital \\ 123 Ta Pei Road \\ Niao Sung Hsiang \\ Kaohsiung 833, Taiwan \\ Phone: 886-7-731-7123, \\ ext. 3399 \\ Fax: 886-7-311-2516 \\ E-mail: \\ brainhemostasis@yahoo.com
}


before $2 \mathrm{pm}$. On that day, he woke up at $5 \mathrm{pm}$ and experienced dizziness, asthenia, unsteady gait with deviation to the left side, and inability to hold objects by hand properly. These discomforts lasted for $30 \mathrm{~min}$ and then spontaneously subsided except for asthenia. He denied any association except generalized perspiration during the attack. Similar episodes recurred 3 to 4 times daily afterwards. Each attack lasted for 10-30 min and then completely recovered. He visited our emergency service 7 days later. He had had a history of hypertension and old cerebral infarction, but only received amlodipine besylate without aspirin for prophylaxis. He lived independently. He denied having foreign travel, craniofacial trauma, flu or infection, unknown drug usage, intoxication, or consumption of herbs, alcohol or cigarettes recently.

On presentation, his vital signs were stable. Physical examination was unremarkable. Neurological examination showed normal speech, higher cortical function, neuro-ophthalmology, cranial nerves, motor system, and sensory function, except for mild intentional tremor of both hands. Cerebellar lesion was interpreted. A stroke risk factor survey, including biochemistry, haematology, lipid profiles, urinalysis, coagulation time, lupus study, antiphospholipid antibodies, immunology, coagulation function, and infection index, was within the reference range, except for hypertriglyceridaemia (260 mg\%; normal: 40-160 mg\%). Head magnetic resonance imaging showed high DWI and low ADC intensities scattered at the left cerebellum, compatible with new infarctions, within the vascular territory of the posterior inferior cerebellar artery (Figures 1A-B). Magnetic resonance arteriogram revealed basilar artery stenosis (Figure 1C). Nerve conduction velocity study did not disclose abnormal findings of peripheral nerves. Left cerebellar infarction was established. Aspirin and nifedipine OROS were prescribed for stroke prevention. He was discharged 6 days later with complete recovery of neurological deficits.

\section{Discussion}

Neurological deficit was found $7 \mathrm{~h}$ after influenza $\mathrm{A} / \mathrm{H} 1 \mathrm{~N} 1$ vaccination in our patient. However, the exact time of onset is actually unknown as he fell asleep unusually for $5 \mathrm{~h}$. The causal relation between vaccination and ischaemic stroke is seriously challenged, similar to previous reports [1-5], especially when our patient does have a few stroke risk factors, such as hypertension, previous stroke, intracranial atherosclerosis, old age, and hypertriglyceridaemia. Our patient's infarction is compatible with small- to medium-size artery occlusion which is also seen in other ischaemic stroke patients after vaccination [2, 5] or vaccination-related angiopathy [8]. Nevertheless, our aim is to highlight a possibility of an underestimation of vascular complications in vaccination, and that would jeopardize an individual who exhibits multiple stroke risk factors as our patient.

Reviewing the literature from PUBMED and BIOSIS, ischaemic stroke has only been reported after vaccination of varicella-zoster virus vaccine $[2,4]$, influenza virus vaccine [5], human papillomavirus vaccine [3], cholera and typhoid vaccine [1], and also HER-2/neu-pulsed dendritic cell vaccine. According to the VAERS [7], the leading vaccine reported to be complicated with ischaemic stroke is seasonal flu, followed by hepatitis A and B virus, pneumococcus, diphtheria/pertussis/tetanus toxoid, poliomyelitis, and Haemophilus influenzae (Figure 2). There are a total of 18 individuals having ischaemic stroke after influenza A/H1N1 vaccination, compared to 164 individuals after seasonal flu vaccination. However, it is the first time of worldwide vaccination with influenza $\mathrm{A} / \mathrm{H} 1 \mathrm{~N} 1$ vaccine. This indicates that ischaemic stroke is not an extremely scarce complication after vaccination. On the other hand, there is only 1 patient who had ischaemic stroke after influenza $\mathrm{A} / \mathrm{H} 1 \mathrm{~N} 1$ vaccination in the United Kingdom [9]. Bias may come from the nature of the reporting system. Nevertheless, it still
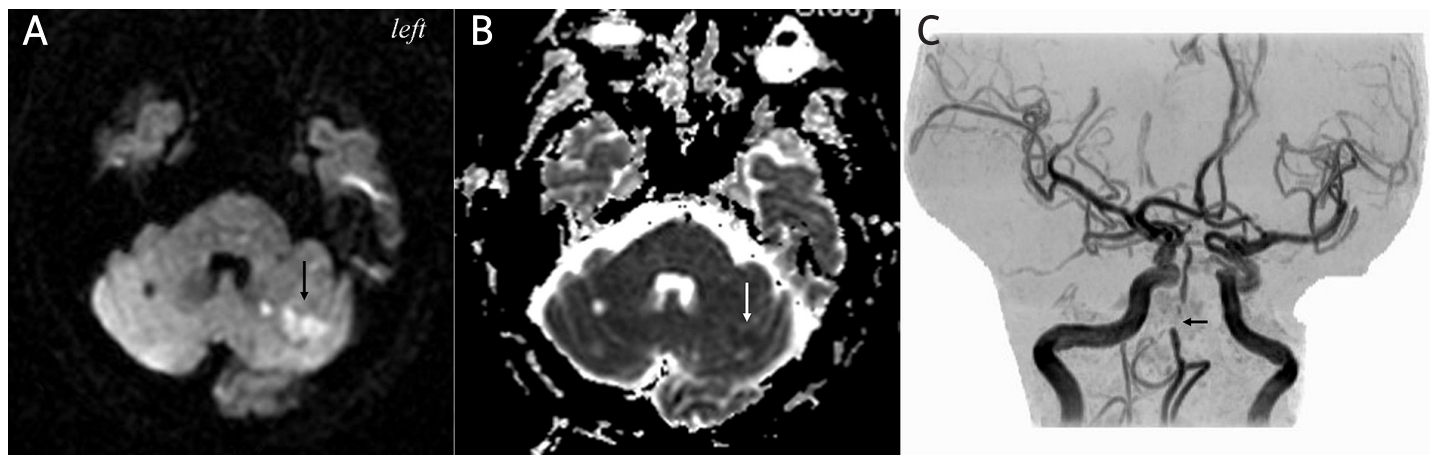

Figure 1. Head magnetic resonance imaging showed scattered lesions exhibiting hyperintensity under DWI (arrow) (A) and hypointensity under ADC sequence (arrow) (B) at left cerebellum compatible with acute infarction. The vascular supply was posterior inferior cerebellar artery. Magnetic resonance arteriogram revealed atheromatous stenosis at basilar artery (arrow) but not distal vertebral artery (C) 


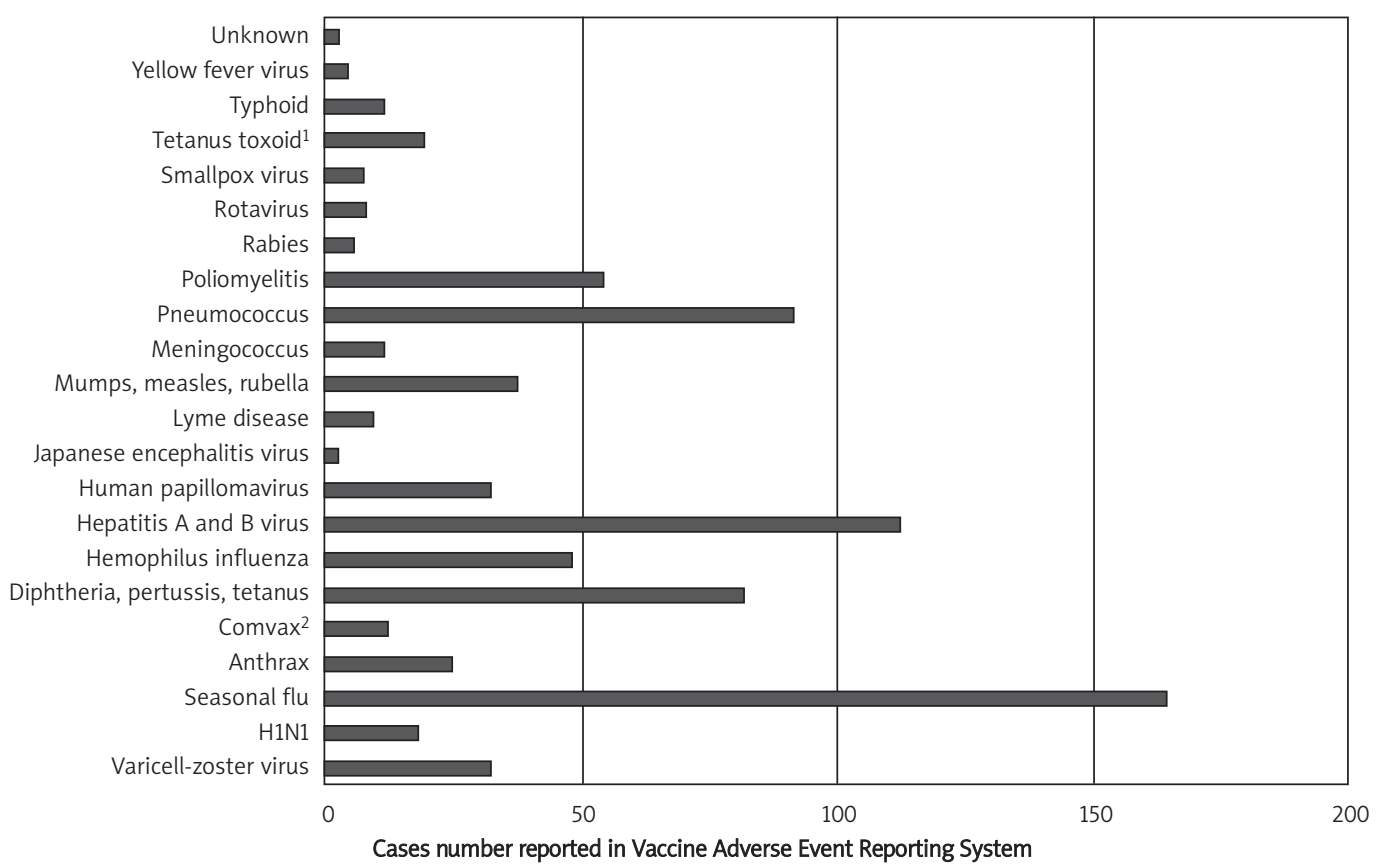

1 Tetanus toxoid - only single tetanus toxoid without other component. Combo vaccine to tetanus toxoid was categoried to other vaccine; 2 Comvax - combo vaccine to hepatitis B virus and Hemophilus influenza only

Figure 2. Frequency of ischaemic stroke reported in various vaccines. The data were extracted from the Vaccine Adverse Event Reporting System (accessed 2010 April 17)

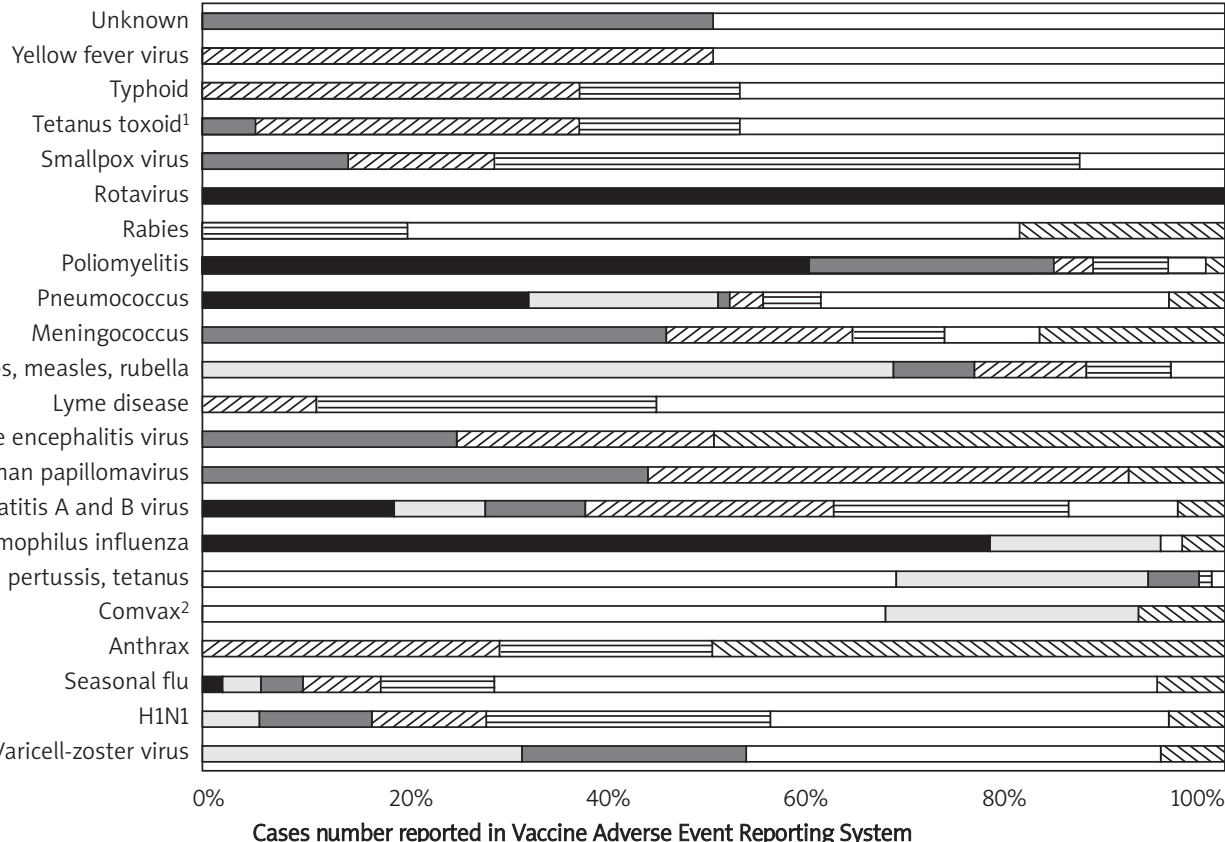
$<1$ year
1-5 years
6-17 years
18-39 years
40-59 years
$\geq 60$ years
Unknown
$\mathbb{\$}$

1Tetanus toxoid - only single tetanus toxoid without other component. Combo vaccine to tetanus toxoid was categoried to other vaccine; 2 Comvax - combo vaccine to hepatitis B virus and Hemophilus influenza only

Figure 3. Proportion of ischaemic stroke events reported according to age groups in various vaccines. The data were extracted from the Vaccine Adverse Event Reporting System (accessed 2010 April 17) 
provides noteworthy resource data to elucidate the trend of frequency and demography of adverse reactions after influenza A/H1N1 vaccination.

Is there any risk factor for ischaemic stroke in vaccination? In fact, statistical analysis is impossible for comparison as the patient sampling from the population is too small. Approximately $38 \%$ and $61 \%$ of ischaemic stroke subjects are over 60 years after influenza $\mathrm{A} / \mathrm{H} 1 \mathrm{~N} 1$ and seasonal flu vaccination, respectively (Figure 3) [7]. The frequency of ischaemic stroke does not significantly increase in children after varicella-zoster virus vaccination [4]. However, nearly half of ischaemic stroke children have concomitant stroke risk factors, such as cardiac diseases, autoimmunity disorders, vasculopathy, or coagulopathy [2-5]. Therefore, vaccination may promote a pre-existing prothrombotic condition. Accordingly, vaccination should be carefully evaluated and monitored in individuals associated with old age or stroke risk factors.

Vaccination can induce inflammatory and immunological responses. A flare-up of lupus has been reported [10]. A generation of temporary inflammatory reactions with an elevation of cytokines after vaccination with Salmonella typhi vaccine, increase of blood antiphospholipid antibodies with influenza vaccine, and vasculitis with a couple of vaccines have been identified. In our patient, we did not detect an abnormal alteration of immune and coagulation function or angiopathy, but that does not completely exclude their participation.

In contrast to a lucid period at least 2 to 9 days for vasculitis and longer in other autoimmune reactions, a rapid onset of symptomatic thrombosis may indicate a hypersensitive reaction in our patients. In fact, ischaemic stroke occurs within the first day in $22 \%$ and $18 \%$ of subjects receiving vaccination with influenza $\mathrm{A} / \mathrm{H} 1 \mathrm{~N} 1$ and seasonal flu vaccine, respectively [7]. Although it is uncertain if an enhancement of inflammatory/immunological activity after vaccination is sufficient for initiating symptomatic vascular occlusion, the risk of an additional prothrombotic effect should be included for consideration of the vaccine programme in future.

\section{References}

1. Brahams D. Damages for stroke after cholera and typhoid vaccination. Lancet 1985; 2: 1372.

2. Wirrell E, Hill MD, Jadavji T, Kirton A, Barlow K. Stroke after varicella vaccination. J Pediatr 2004; 145: 845-7.

3. Borja-Hart NL, Benavides S, Christensen C. Human papillomavirus vaccine safety in pediatric patients: an evaluation of the Vaccine Adverse Event Reporting System. Ann Pharmacother 2009; 43: 356-9.

4. Donahue JG, Kieke BA, Yih WK, et al. Varicella vaccination and ischemic stroke in children: is there an association? Pediatrics 2009; 123: e228-34.
5. Vainer-Mossel ED, Mekori YA, Mor A. Ischemic stroke in a patient with lupus following influenza vaccination: a questionable association? Isr Med Assoc J 2009; 11: 186-7.

6. Urbanek C, Palm F, Grau AJ. Influenza and stroke risk: a key target not to be missed? Infect Disord Drug Targets 2010; 18: 9.

7. VAERS: Vaccine Adverse Event Reporting System. From: http://wonder.cdc.gov/vaers.html (accessed 2010 April 17).

8. UK Suspected Adverse Reaction Analysis. Swine Flu (H1N1) Vaccines - Celvapan and Pandemrix. From: http://www.mhra.gov.uk (accessed 2010 April 17).

9. Agmon-Levin N, Kivity S, Shoenfeld Y. Influenza vaccine and autoimmunity. Isr Med Assoc J 2009; 11: 183-5.

10. Kelsall JT, Chalmers A, Sherlock CH, Tron VA, Kelsall AC. Microscopic polyangiitis after influenza vaccination. J Rheumatol 1997; 24: 1198-202. 